Itinéraires Itinéraires

Littérature, textes, cultures

\title{
What's in a Name : la masculinité à l'épreuve de Shakespeare relu par Wilde
}

\section{Gilbert Pham-Thanh}

\section{(2) OpenEdition}

\section{Journals}

\section{Édition électronique}

URL : http://journals.openedition.org/itineraires/1702

DOI : $10.4000 /$ itineraires. 1702

ISSN : 2427-920X

Éditeur

Pléiade

\section{Édition imprimée}

Date de publication : 1 décembre 2010

Pagination : 45-58

ISBN : 978-2-296-13183-5

ISSN : 2100-1340

\section{Référence électronique}

Gilbert Pham-Thanh, "What's in a Name : la masculinité à l'épreuve de Shakespeare relu par Wilde», Itinéraires [En ligne], 2010-4 | 2010, mis en ligne le 01 décembre 2010, consulté le 26 avril 2019. URL http://journals.openedition.org/itineraires/1702 ; DOI : 10.4000/itineraires. 1702

\section{(c) $\oplus \Theta \Theta$}

Itinéraires est mis à disposition selon les termes de la licence Creative Commons Attribution - Pas d'Utilisation Commerciale - Pas de Modification 4.0 International. 


\title{
What's in a Name : la masculinité à l'épreuve de Shakespeare relu par Wilde
}

\begin{abstract}
Wilde revisits Shakespeare's Sonnets to hint at their homoerotic contents. His short story, The Portrait of Mr.W. H., is an occasion to promote a form of attachment between male late Victorians who try to crack the identity of the poems' male dedicatee. More generally, it is the constructedness of masculinity that Wilde makes it possible to formulate, in a synthetic approach to both masculinity and literature. In consequence, his investigation into the Shakespearean poems contributes to positing the textual nature of masculinity, and therefore its fundamental openness to intervention.
\end{abstract}

Keywords : essence-construction, homoerotic, homosocial, masculinity, Wildean critic as artist

Mots clés : essence-construction, homoérotique, homosocial, masculinité, le critiqueartiste wildien

L'analyse tente d'articuler trois temporalités, puisqu'elle reprend, depuis le présent des études des masculinités, l'interprétation qu'Oscar Wilde donnait dans le contexte fin-de-siècle des Sonnets du Barde élisabéthain de Stratford-on-Avon. Évoquer la modernité des deux poètes-dramaturges amènera à chercher des intersections entre périodes qui, c'est le parti pris épistémologique, partagent un certain nombre de paradigmes. En outre, ce travail se place sous l'influence au moins partielle de la réflexion wildienne sur l'activité critique, dont on peut dès à présent noter qu'elle surplombe la création poétique et dramatique de Shakespeare. Elle reconfigure en effet le recueil de poèmes dans des thématiques et des problématiques modernes, tout en réinscrivant l'éloge versifié de l'amant mystérieux au sein d'une forme narrative moderne, la nouvelle de détection. Il appartient toutefois à un geste critique extradiégétique, et plus tardif encore, de mettre en lumière certains pans hyper-modernes de l'étude fictionnelle que Wilde propose des vers de Shakespeare. 
Les cent cinquante-quatre sonnets demeurent certes une référence obligée, en tant que source primaire de The Portrait of $M r . W . H .(P W)$, mais c'est cette nouvelle qui constitue le corpus principal, dans sa version courte de 1889 . Isobel Murray ${ }^{1}$ en souligne à juste titre la composition mieux maîtrisée, par rapport à celle de 1921. Wilde y reprend au travers des exégèses de trois personnages, le narrateur, George Erskine et Cyril Graham, l'hypothèse déjà avancée au XVIII siècle par Thomas Tyrwhitt ${ }^{2}$ concernant l'identité du dédicataire des fameux Sonnets publiés en 1609. Selon lui, il s'agit de William Hughes, jeune acteur qui fait partie de la troupe de Shakespeare et exerce sur son aîné une influence profonde, y compris dans sa production artistique. Cyril commande un tableau, qu'il présente comme un authentique portrait du jeune élisabéthain, afin d'accréditer frauduleusement cette théorie. À cette occasion, et reprenant des éléments de « The Truth of Masks » ou de « The Decay of Lying ${ }^{3}$ », Wilde interroge la valeur de la contrefaçon, moralement considérée comme un déni du réel, peut-être en tant qu'acte contre-nature. Il en sonde en particulier les possibilités heuristiques, notamment dans la construction des identités sexuelles.

La recherche sur cette dernière thématique, en contextes élisabéthain et victorien, est fertile. On se bornera à souligner la façon stratégique dont Wilde se concentre sur les inscriptions d'une nébuleuse homosociale, en particulier dans les cent vingt-six premiers sonnets, à l'occasion de son enquête sur les modalités d'expression d'une subjectivité masculine hétérodoxe. L'investigation relative à l'identité du mystérieux Mr. W. H. introduit donc le lecteur à un univers où l'identité virile se recompose. Bien qu'elle neutralise la prégnance du scénario hétérosexuel dans les poèmes, l'intervention wildienne ne trahit pas Shakespeare en y explorant les amours masculines. Elle reprend l'inscription poétique d'un fait historique socialement acceptable, voire louable, qui place dans l'amour chevaleresque entre frères d'armes un idéal quasi spirituel, afin de l'assimiler à une habitude mal définie, devenue de plus en plus suspecte aux yeux d'une époque moderne dont Michel Foucault montre le caractère disciplinaire et répressif ${ }^{4}$. C'est la force de la proposition wildienne que de s'emparer de l'image du Barde, véritable institution nationale, afin d'en faire le champion d'une cause contestable au XIX ${ }^{\mathrm{e}}$ siècle. Dans ce but, Wilde fait jouer niveaux de réalité et régimes discursifs divergents, postulant dans l'unité du texte leur solidarité fondamentale, voire leur caractère interchangeable.

1. Oscar Wilde, Complete Shorter Fiction, Introduction, Oxford, Oxford University Press, 1979, p. 12.

2. Lettre à Edmond Malone rapportée dans Supplement to the Edition of Shakespeare's Plays, 1778.

3. «The Truth of Masks » [1885], « The Decay of Lying » [1889], Collected Works of Oscar Wilde, Ware, Wordsworth, 1997, p. 873-893, p. 775-799.

4. Michel Foucault, Surveiller et punir [1975], Paris, Gallimard, 1985. 
C'est tout d'abord la situation d'énonciation de The Portrait of $M r$. $W$. H. qui introduit la thématique de l'homosocialité, décrivant un phénomène trans-historique assez commun dans une Grande-Bretagne qualifiable de patriarcale, en dépit des règnes d'Élisabeth $\mathrm{I}^{\mathrm{re}}$, sous lequel Shakespeare s'illustre, et de Victoria, qui voit Wilde s'imposer. Le narrateur, son ami George Erskine, auquel il rend visite, et Cyril Graham, longuement évoqué, composent un paradigme androcentré. Ces trois célébrants communient autour de la double figure totémique de Mr. W. H. et de Shakespeare, eux-mêmes symboles de la proximité fusionnelle entre hommes chantée au sonnet 42 : «But here's the joy, my friend and I are one ${ }^{5}$. » Ce dispositif phallocratique se complète des références au monde de la domination masculine, la Public School d'Eton (PW I : 141), l'université de Cambridge et son association d'art dramatique, l'Amateur Dramatic Company ( $P W \mathrm{I}$ : 143), ainsi que le club londonien où le narrateur apprend les intentions suicidaires d'Erskine ( $P W$ III : 167). Quant aux poèmes de Shakespeare qu'ils récitent et relisent inlassablement, ils mettent d'habitude en scène les relations homosociales unissant la persona à ce jeune homme blond évoqué au sonnet $144^{6}$, à qui il ne manque plus qu'un fils, comme le suggère le sonnet 13 : «You had a father, let your son say so ${ }^{7}$ » $(P W \mathrm{I}: 145)$. La société que Wilde dépeint demeure patrilinéaire.

Cette hégémonie ne laisse aux figures de la femme qu'une place réduite. Si la Dame en noir n'apparaît dans les Sonnets qu'à l'occasion ${ }^{8}$, elle n'inspire plus rien à un Wilde qui se borne à l'identifier en la personne de Mrs. Mary Fitton dès les premières pages ( $P W \mathrm{I}: 141)$. Deux autres figures féminines dépourvues de toute charge érotique sont introduites, Lady Erskine, mère de George ( $P W$ III : 168), et la femme anonyme de l'auteur du fameux portrait, Edward Merton, qui est rendue à l'insignifiance en quelques mots : «I $[\ldots]$ found a pale, interesting young man, with a rather common-looking wife ${ }^{9} »(P W \mathrm{I}: 151)$.

Cependant, la référence à une communauté masculine soudée renforce moins l'organisation patriarcale de la société qu'elle n'organise l'expression d'une sensibilité qualifiable d'homoérotique. Les Sonnets donnent l'exemple de cet homme ouvrant son cœur, pris dans des logiques d'investissement de la sphère du sentiment. Wilde reprend des vers où l'utilisation de figures refuse la rigueur virile de la littéralité, et où le recours au lexique

5. «Mais, joie! de lui et moi comme un seul est formé » (les traductions des Sonnets sont dues à André Mansat, Les Sonnets de Shakespeare, Paris, Didier, 1970).

6. " The better angell is a man right faire » (« le bon ange est un homme, et blond absolument $»)$.

7. « $[\mathrm{T}] \mathrm{u}$ connus ton parent; / veuille, alors, que ton fils, un jour, en fasse autant ».

8. Sonnets 127 et 144 , de manière évidente.

9. « Je trouvai $[\ldots]$ un fort intéressant jeune homme au teint pâle, en compagnie de sa femme, personne à l'apparence plutôt ordinaire. » 
de la délicatesse offre à la persona du sonnet 79 l'occasion de confesser une dépendance affective qui trahit un manque de contrôle de soi :

Whilst I alone did call upon thy aid,

My verse alone had all thy gentle grace

But now my gracious numbers are decayed,

And my sick Muse does give another place ${ }^{10}$;

Tant de lyrisme rompt avec l'idéal guerrier et renvoie au modèle du courtisan, à l'aise dans ces contextes élégants où évoluent aussi les héros wildiens. Ces hédonistes raffinés sont présentés dans la bibliothèque d'Erskine, où ils devisent en buvant du café, une cigarette à la main. La scène se structure en tableau de la décomposition complaisante, où les volutes de fumée contestent les lignes droites, le culte de la production et de la substance, créant une atmosphère atemporelle d'oisiveté méditative : « Erskine remained silent for a few moments, looking at the thin grey threads of smoke that were rising from his cigarette ${ }^{11} »(P W \mathrm{I}: 139)$. Ce cadre n'est pourtant qu'un fond sur lequel se dessine une présence masculine en gloire, louée par une voix et une focalisation masculines elles aussi, puis reprise à l'intérieur du projet littéraire d'un homme de lettres, tout concourant à la construction d'une érotique où les regards croisent les chairs et creusent les sentiments. Reprenant dans le sonnet 105 le thème de l'amour refusé à Dieu, Shakespeare fait allusion au culte des idoles afin de traduire la force de son émoi :

Let not my love be called Idolatrie,

Nor my beloved as an Idoll show,

Since all alike my songs and praises be

To one, of one, still such, and ever so ${ }^{12}$.

Il est vrai que la persona détecte dans l'apparence physique du jeune homme la réalisation de l'idéal masculin. Wilde reprend cette notion d'idolâtrie, devenue folle, «mad idolatry » ( $P W$ II : 161), pour décrire l'attitude de la persona au sonnet 93, et lorsqu'il évoque le prétendu portrait de Hughes, offre une variante du blason, occasion de détailler les attraits de sa physionomie : « its dreamy wistful eyes, and its delicate scarlet lips ${ }^{13}$ » ( $P W \mathrm{I}$ : 140). La thématique se dissémine par la création du beau Cyril,

10. «Aussi longtemps que seul j'invoquais ton appui, / seul mon poème aussi se paraît de tes grâces; / mes vers dégénérés n'ont de charme aujourd'hui, / et ma Muse malingre à une autre fait place; $»$.

11. «Pendant un long moment, Erskine restait silencieux, regardant les minces filets de fumée grise qui s'élevaient de sa cigarette. »

12. "Ne dites pas "Idolâtrie!" de mon amour, / ne dites pas : "c'est une idole!" de qui j'aime, / même alors que mes chants, mes louanges, toujours / vont vers lui, sont pour lui, et sans cesse les mêmes! »

13. «[S]es yeux rêveurs et mélancoliques, et ses délicates lèvres écarlates ». 
spectateur enthousiaste de sa propre personne : « he was very languid in his manner, and not a little vain of his good looks [...]. He certainly was wonderfully handsome ${ }^{14} »(P W I: 142)$. Le narcissisme se double du voyeurisme d'Erskine, de Wilde et du lecteur, en un réseau textuel qui se complète de la persona shakespearienne et du narrataire, à qui il est demandé de s'admirer dans les vers éternels des poèmes, au sonnet 18 :

But thy eternall Sommer shall not fade,

Nor lose possession of that faire thou ow'st ${ }^{15}$,

La topique narcissico-voyeuriste érotise un peu plus le tableau de la masculinité et l'évocation du corps du jeune homme aimé.

Il est toutefois difficile de parler de sexualité, du moins dans le cas de Shakespeare, puisque l'amour ne trouve pas dans la sphère des contacts génitaux ses modalités d'expression. Le sonnet 20 est d'ailleurs sans ambiguitté, car la persona voit dans l'attribution d'un pénis à l'être aimé une raison de conserver ses distances :

And for a woman wert thou first created,

Till nature as she wrought thee fell a dotinge,

By adding one thing to my purpose nothing ${ }^{16}$.

Dans ce sentiment encore mal défini, Wilde croit déceler les signes d'un attrait charnel, hypothèse qu'il appuie sur les jeux de mots récurrents rapprochant Hughes et use, « utiliser » ( $P W \mathrm{I}: 148)$. En effet, Francis Meres est convoqué par Wilde pour rappeler que son contemporain Shakespeare destine ses poèmes à une communauté de lecteurs choisis, "as Meres tells us, for private circulation only among a few, a very few, friends ${ }^{17}$ » $(P W$ II : 157), lecteurs à même de comprendre certains messages codés empruntés au paradigme gay ${ }^{18}$. Si Wilde ne renonce pas aux circuits de grande diffusion, il puise dans la langue de la fin du XIX ${ }^{e}$ siècle, quant à lui, les chiffres de l'homoérotisme. Ainsi, l'accumulation de vocables tels «fascinated » $(P W \mathrm{I}: 140 ; \mathrm{III}: 166)$, « effeminate » $(P W \mathrm{I}: 140,142)$, « wonderful» $(P W \mathrm{I}: 140,141,148)$, «strange » $(P W \mathrm{I}: 141,146)$, « delightful» $(P W \mathrm{I}: 141)$, « languid» $(P W \mathrm{I}: 142)$ ou $«$ musically ${ }^{19} »$

14. « [S]es manières étaient pleines de langueur, et il ne se montrait pas peu vain de son apparence avantageuse [...]. C'était un garçon merveilleusement beau, à n'en point douter. » 15. «Ton éternel été ne saurait passer, lui, / ni se perdre ce bien, la beauté qui est la tienne, $»$.

16. «Et c'est femme d'abord que Nature t'œuvra, / Mais, saisie d'un accès d'amoureux radotage, / Elle ajouta un rien qui de toi m'évinça ».

17. « [C]omme l'indique Meres, destinés à une diffusion privée, au sein d'une communauté d'amis choisis, très choisis. »

18. L'utilisation de l'adjectif «homosexuel» situe le discours dans le registre médicorépressif, et sera évité.

19. «[F]asciné », « efféminé », merveilleux », « étrange », « délicieux », « languissant », 
( $P W$ III : 159) fait plus que renvoyer à l'esthétisme. Même si elle reste allusive, l'interprétation que Wilde donne de la relation entre Shakespeare et son narrataire est sans ambiguïé :

Who was that young man $[\ldots]$ who $[\ldots]$ was addressed by him in terms of such passionate adoration that we can but wonder at the strange worship, and are almost afraid to turn the key that unlocks the mystery of the poet's heart? ${ }^{20}$ (PW I : 146)

Une voix dissidente est mise au service d'une focalisation qui surdétermine certains détails, comme dans cette banale indication : " Erskine [...], a man of forty, suddenly put his hand upon my shoulder ${ }^{21} »(P W \mathrm{I}$ : 139). Les corps sont mis en jeu, le « je»s'incarne afin de construire par le contact physique un «nous » complice. Dans les marges de la diégèse, s'inscrit alors en filigrane le récit d'une masculinité alternative, qui s'efforce de trouver ses propres régimes signifiants afin d'apporter des éléments de réponse à cette question lancinante et troublante posée par l'hérö̈ne de Romeo and Juliet et revue par Lord Alfred Douglas, l'amant de Wilde : " what's in a love that dare not speak its name ${ }^{22}$ ?"

Certes, le sentiment homophile ne marque pas seulement une réaction à l'ordre patriarcal hégémonique, mais il doit tout de même être replacé dans une configuration élargie qui prend en compte sa valeur polémique. Cette contestation de l'hétéronormativité remet en question l'idéologie dominante, tout en élaborant une esthétique de la rupture qui met en tension lecture, interprétation et réécriture des Sonnets. C'est en particulier la manière dont Wilde permet de redéfinir la notion de texte qui doit être évoquée, comme préalable au dernier moment de l'analyse de la masculinité.

The Portrait of Mr. W. H. est avant tout un texte hybride, qui compte une trentaine de citations longues, toutes insérées en pavé, auxquelles s'ajoutent les vers intégrés dans le corps du texte. Cette œuvre citationnelle, hantée par la présence de Shakespeare, rend la notion d'auteur problématique, comme dans ce passage remarquable où la phrase se déploie, entièrement portée sans signaux démarcatifs par les sonnets 20,26, 126, $109,1,2,8$, et 22 :

Who else but he could have been the master-mistress of Shakespeare's passion, the lord of his love to whom he was bound in vassalage, the delicate minion of pleasure, the rose of the whole world, the herald of the spring, decked in the proud livery of youth, the lovely boy whom it was sweet

« musicalement ».

20. «Qui était ce jeune homme [...] à qui [...] il s'adressait en termes d'adoration passionnée tels que l'on doit s'interroger sur une adoration aussi étrange, qui nous fait presque redouter de tourner la clé déverrouillant le mystère du cœur du poète. »

21. «Erskine, un homme âgé de quarante ans, mit soudain sa main sur mon épaule.»

22. «Qu'y a-t-il dans cet amour qui n'ose pas dire son nom?» 
music to hear, and whose beauty was the very raiment of Shakespeare's heart, as it was the keystone of his dramatic power ${ }^{23}$. (PW II : 159)

La poésie shakespearienne est tissée dans le texte wildien, et la diégèse s'appuie sur elle pour faire progresser l'enquête, repérant, dans tel vers du sonnet 94, l'indice révélant que Mr. W. H. fait bien partie de la communauté des acteurs : " That do not do the thing they most do show, / Who, moving others, are themselves as stone ${ }^{24} »(P W$ II : 161). Tronqués, les poèmes sélectionnés sont ainsi réintégrés dans un récit qui transpose la dimension dramaturgique initiale du recueil dans le déroulement de l'intrigue.

Cette composition morcelée de la nouvelle fait écho à celle du recueil de sonnets, qui met en tension une esthétique de la forme brève à la perfection formelle, donc autosuffisante, renforcée par le distique final placé en retrait, et d'autre part une rhétorique fragmentaire, cumulative, ou sérielle, voire une poétique proliférante, qui traverse les cent cinquantequatre poèmes. L'objet est à la fois parfaitement dessiné en quatorze vers, alors qu'une série longue mime l'effort vain d'atteindre une complétude donnée comme illusoire par la démultiplication des perspectives. Ainsi, la macro-structure du recueil replace chaque poème dans une logique de la défaillance, qui trouve un point d'application jusque dans l'unité fine du sonnet 126, amputé du distique de clausule et ne comptant que douze vers.

En regard, le texte wildien paraît d'abord bien lisse. Résolument romanesque, il décrit les relations de trois personnages, où mystères, investigations, morts violentes et supercheries dynamisent une histoire cosmopolite, dont l'aspect parfaitement cyclique transparaît dans les marqueurs spatiaux, qui déplacent la scène de Londres $(P W \mathrm{I}: 143)$ vers l'Allemagne, puis la France ( $P W$ III : 167), et enfin l'Angleterre ( $P W$ III : 168). En fait, la narration semble conduite comme un récit de détection, mais Wilde ne recourt à cette tradition que pour élaborer son propre mode de composition hybride.

En effet, la trame poético-romanesque se tisse avec celle de la recherche érudite, à laquelle elle emprunte ses protocoles. Elle plonge dans les documents, convoque les experts, « a German commentator called Barnstorff $^{25} \gg(P W 1: 146)$, ou encore Francis Meres ( $\left.P W \mathrm{I}: 144\right)$, vérifie

23. "Qui d'autre que lui aurait pu être maître-maîtresse de la passion de Shakespeare, le seigneur de son amour, lui à qui son devoir fut lié par son mérite en étroit vasselage, chéri de ses faveurs, la rose du monde tout entier, le héraut du printemps, paré de ce fier habit, ta jeunesse, l'adorable garçon dont si musique est l'ouïr, dont la beauté n'est que le vêtement seyant que porte le cœur de Shakespeare, puisqu'il était la clef de voûte de sa puissance dramatique. »

24. «[N]e faire pas ce que le plus prétend faire, / mouvoir le cœur d'autrui, et rester médusé ».

25. « $[\mathrm{U}] \mathrm{n}$ commentateur allemand du nom de Barndorff. » 
les données, qu'elle organise ensuite : « for three months we did nothing but go over each poem line by line, till we had settled every difficulty of text or meaning ${ }^{26} \gg(P W \mathrm{I}: 150)$. Cette autorité savante ne parvient pourtant ni à établir les faits ni à réguler la narration. Si Wilde s'en réclame afin de montrer le sérieux de sa démarche, il adopte tout autant une approche parodique, et retrouve ainsi des logiques littéraires. En conséquence, se dégage ici la notion wildienne d'une critique créative issue d'un critique démiurge, dont les contours sont définis dans "The Critic as Artist ${ }^{27}$ ». La démonstration perd sa rigueur géométrique lorsque la construction du sens s'infléchit de façon subjective assumée, voire revendiquée, ce qu'annonce le choix de composer dans un genre fictionnel. Vers la fin de la nouvelle, l'auteur fait significativement résonner son nom de manière homophonique : «I said all kinds of wild things ${ }^{28}$ » ( $P W$ III : 168), mais surtout, il utilise des procédures d'attestation modales, introduites par des adverbes, comme dans « obviously [...], of course obvious » (PW I : 148) ou encore « Perhaps $[\ldots]$, surely » $(P W \text { II : 163 })^{29}$.

Ailleurs, le registre prend une dimension mystico-religieuse, lorsque le récit célèbre un rituel qui s'apparente parfois à une confession de foi : «I believe in Willie Hughes ${ }^{30} »(P W \mathrm{I}: 152)$. La triple occurrence du verbe " croire » en quelques lignes ( $P W \mathrm{I}: 141)$ modifie la nature du texte de façon radicale, et constitue un paradigme structurant dans lequel d'autres termes se rangent, « converted » $(P W \mathrm{I}: 141)$, « martyrs » $(P W \mathrm{I}: 152)$ et « faith» $(P W$ III : 167), infléchissant le sens de « devoted» $(P W \mathrm{I}: 142)$, «adoration» $(P W \mathrm{I}:$ 146), «sacrificed» $(P W \mathrm{I}: 153)$ ou «spiritual» $(P W \text { II : 159 })^{31}$. Tous les registres s'unissent pour créer un régime signifiant spécifique qui construit une réalité située dans un espace singulier, bien que pluriel, faisant dialoguer des traditions différentes par l'entremise de la pensée paradoxale de Wilde. Ainsi, la recherche de l'identité de Mr. W. H., qui métaphorise peut-être la volonté d'explorer l'énigme de l'homophilie, montre qu'elle est capable de générer bien des types de discours susceptibles d'être tissés harmonieusement pour répondre à l'interrogation angoissée d'un Coriolan placé sous l'influence du Lord Henry de The Picture of Dorian Gray: «there is a world winged with paradoxes, elsewhere ${ }^{32}$ !»

26. « $[T]$ rois mois durant, nous ne fîmes rien que reprendre chaque poème, ver après ver, jusqu'à pouvoir résoudre chaque difficulté posée par le texte ou par sa compréhension. »

27. «The Critic as Artist» [1890], Collected Works of Oscar Wilde, Ware, Wordsworth, 1997, p. 819-872.

28. « J'ai dit toutes sortes de choses fort déraisonnables/à la wilde ».

29. «Évidemment [...], bien sûr », « Peut-être [...] assurément ».

30. «Je crois en Willie Hughes».

31. « [C]onverti », « martyrs », « foi », « dévoué », « adoration », « sacrifié », « spirituel ».

32. «Il y a un monde porté par les ailes du paradoxe, ailleurs!» 
La fascination que les héros wildiens expriment les uns pour les autres comme pour le William Hughes du portrait, rappelle l'attrait puissant de Shakespeare à l'égard du jeune homme des poèmes. Ce réseau trace dans The Portrait of Mr. W. H. les limites d'une sphère homophile. L'expression hyperbolique de l'affect invite cependant à ne pas considérer l'objet des louanges en tant que personne réelle, comme l'indique le sonnet 93, cité par Wilde : « But heaven in thy creation did decree, / That in thy face sweet love should ever dwell ${ }^{33} \gg(P W$ II : 161). Un travail d'idéalisation s'opère, étayé par l'utilisation de l'idiome religieux déjà noté. Le mécanisme verbalise l'aspiration vers l'amant parfait ou bien la quête d'un idéal du moi. Dans ces deux cas, il s'agit d'élaborer une figure masculine fantasmée capable d'orienter sentiments et comportements, ou plus exactement, dans la perspective à présent retenue d'une critique de type wildien, de repérer dans la construction d'une fiction l'effort de produire une définition de la masculinité elle-même, à travers l'une de ses manifestations stylisées. Il faut aller plus loin en posant que Wilde, lorsqu'il compose un texte inclassable, dessine une masculinité inclassable elle aussi. L'anecdotique prend valeur exemplaire et métaphorise une réflexion qui dépasse la seule défense de l'identité gay, s'appuyant sur toutes les procédures de mise en texte, qui contestent la notion d'une réalité fondamentale donnée comme naturelle. La masculinité se déconstruit et laisse place à une identification queer, qui s'inspire d'une approche constructiviste de la personne.

Ce sont les genres sexués, censés livrer l'essence même de la personne, que Wilde met d'abord en perspective. Il note stratégiquement l'habitude prise par les dramaturges de composer certains rôles féminins en pensant à des acteurs précis. Ainsi, Cyril voit en William Hughes la matrice de Viola, Imogène, Juliette, Rosalinde, Portia, Desdémone et même Cléopâtre $^{34}$ (PW I : 147). Ce transformisme quasi transsexuel déplace la notion de modernité, en marge des études gay et d'une conception postmoderne de la littérature.

L'obligation, sous Élisabeth Ire, de faire jouer les rôles féminins par des jeunes hommes se lit ici comme déstabilisation des genres sexués. Elle cautionne et indirectement promeut un régime de visibilité alternatif, où l'homme peut légitimement porter un costume de femme, forme de cross-dressing, et tenir dans l'échange intersubjectif le rôle conventionnel féminin, inscrivant dans la tradition la possibilité d'une telle individuation paradoxale. Wilde repère d'ailleurs la persistance de cette habitude dans le monde victorien, indiquant l'interdiction faite aux femmes de monter sur les scènes de l'université de Cambridge ( $P W \mathrm{I}: 143$ ), bien que dans la même société et au même moment, des actrices incarnent dans les

33. « Mais il fut décrété par le Dieu créateur / que l'amour à jamais vivrait dans ton visage ».

34. Twelfth Night, Cymbelene, Romeo and Juliet, As You Like It, The Merchant of Venice, Othello, Anthony and Cleopatra. 
théâtres des personnages masculins. Le fait sociétal est pourtant transfiguré lorsqu'Erskine déclare n'avoir jamais vu l'égal de Cyril dans le rôle de Rosalinde : « Cyril was the only perfect Rosalind I have ever seen. It would be impossible to describe to you the beauty, the delicacy, the refinement of the whole thing ${ }^{35}$ » (PW I : 143). Le terme thing, " chose », marque le refus de fixer l'identité genrée de Cyril, bien que chez Shakespeare, il soit davantage question d'une ouverture aux deux identifications qu'à leur rejet, comme l'atteste le sonnet 53 :

Describe Adonis and the counterfeit,

Is poorely immitated after you,

On Hellens cheeke all art of beautie set,

And you in Grecian tires are painted new ${ }^{36}$ :

L'individu peut jouer indifféremment les rôles masculins ou féminins, simples masques. En conséquence, il est dans l'ordre des choses qu'un garçon interprète Rosalinde, jeune fille qui doit, dans As You Like $I t$, se déguiser en homme, Ganymède, qui devra à son tour se déguiser en jeune fille. Jubilatoire et iconoclaste, ce tourbillon des genres sexués offre la possibilité même d'une existence festive qui ne rompt pas avec les conventions mais s'en joue. En définitive, Wilde, autant que Shakespeare, loue la consistance de la représentation de soi, qui implique une mise en scène de soi, et génère du réel, dans un univers fondamentalement procédural. Féminin et masculin demeurent des modalités d'inscription dans une société répressive qui catégorise et sépare, mais progressive aussi, puisqu'elle s'accommode des manipulations d'individus mal à l'aise dans les définitions institutionnelles.

La thématique de la construction des genres est reprise dans une problématique plus générale de la constitution des identités. Si Wilde cite ce même sonnet 53 ( $P W$ II : 154), c'est parce que le narrataire y est identifié à un acteur, statut qui lui donne sa seule substance connaissable et son pouvoir d'attraction. Séduit par l'équation shakespearienne entre d'une part le sujet et d'autre part le jeu de masques, servi dans le poème élisabéthain par un jeu de mots sur shaddowes, qui rapproche " ombre » et « rôle », il s'approprie la tension entre monstration, dissimulation et auto-engendrement caméléonien :

What is your substance, whereof are you made,

That millions of strange shaddowes on you tend ${ }^{37}$ ?

35. « Cyril est l'unique Rosalinde parfaite que j'ai jamais vue. Il serait impossible de vous décrire sans rien omettre la beauté, la délicatesse, le raffinement de son interprétation / la chose. » 36. « Décrit-on Adonis? c'est toi représenté / dans la pâle copie que l'image nous donne, / et, si l'on met au front d'Hélène art et beauté, / sous des vêtements grecs, c'est peindre ta personne : ». 37. «Toi qu'escortent d'étranges ombres par milliers, / de quoi donc es-tu fait, quelle est donc ta substance?» 
L'impossibilité de définir la substance du narrataire révèle une individualité qui se dé-substantialise, alors que la persona chante la réalité protéiforme du jeune homme. Cette plasticité anti-essentialiste fonde le sujet queer, dont l'existence phénoménologique ne précède aucune essence mais en marque le déni.

Wilde n'est cependant pas si radical, lorsqu'il se contente d'insister sur le caractère efféminé de Cyril ( $P W \mathrm{I}: 2)$, tout autant que du joli garçon peint à la manière de François Clouet $(P W \mathrm{I}: 139)$ ou du narrataire du sonnet 20 :

\section{A womans face with natures owne hand painted, Hast thou the Master Mistris of my passion ${ }^{38}$,}

Dans The Portrait of Mr.W. H., il ne conserve de ce poème que l'allusion finale à la relation de servitude ( $P W$ II : 159). Il s'accorde cependant avec Shakespeare pour célébrer la confusion des déterminants de genre, qui se détachent des prescriptions sociales fondées sur une représentation idéologique d'un supposé ordre naturel. Ainsi, l'homme se construit en s'attribuant des caractères spécifiquement féminins, c'est-à-dire exclusifs du masculin, qui se voient alors déliés d'une origine biologique.

Le corpus fait en outre émerger la notion d'une masculinité considérée comme grand récit, à la fois narration sociale contrôlant les comportements, et texte pris en charge par l'individu souverain. Le processus est progressif, indirect. Parce que le narrataire idéal est identifié à Mr. W. H., celui-ci devient le signifiant bien réel d'un signifié fantasmé. Pourtant, le rapprochement frappe pour ainsi dire le dédicataire d'irréalité, renvoyant alors son alter ego poétique à sa sphère fictive natale. Tous deux tracent implicitement les contours d'une masculinité assez peu organique, fondée dans le texte littéraire qui en livre le portrait. Ces ombres métaphorisent l'impossibilité de définir ce qu'est un homme, sinon au travers d'une écriture allusive, d'un récit figuré et d'une fiction lacunaire. La nouvelle suggère en effet longtemps que c'est à partir des seuls Sonnets, dont Jean Fuzier évoque l'obscurité ${ }^{39}$, qu'il est possible de définir Mr. W. H., par exemple lorsque Cyril insiste pour ignorer tout élément externe aux poèmes, "working purely by internal evidence ${ }^{40}$ » ( $P W \mathrm{I}$ : 143). Ainsi, la structure creuse des initiales ne prend consistance que dans le texte, lorsque l'exégète détermine le prénom du narrataire en se référant évasivement au jeu de mots qui structure le sonnet 135. La démonstration est convaincante, puisque l'utilisation des majuscules et des italiques dans le poème suggère une lecture à double niveau :

38. «Un visage de femme, et par Nature peint, / Tu l'as, de ma passion, O toi, maîtremaîtresse! »

39. Jean Fuzier, Les Sonnets de Shakespeare, Paris, Armand Colin, 1970, p. 11.

40. « [M]'appuyant uniquement sur des preuves internes [...].» 
So thou beeing rich in Will adde to thy Will,

One will of mine to make thy large Will more ${ }^{41}$.

Si Wilde ne cite pas ce poème, il en va différemment lorsqu'il déduit le nom Hughes d'un autre jeu de mot fondé sur l'homophonie, qui rapproche le prénom et hews, «des nuances », dans le sonnet 20 cette fois : «A man in hew all Hews in his controwling ${ }^{42}$. » En d'autres termes, William Hughes existe pour les personnages wildiens parce qu'ils le trouvent dans le recueil, sa représentation et sa désignation scripturale valant comme preuve et détermination de sa réalité. Il prend vie par sa seule nature verbale, comme le suggère le narrateur de The Portrait of Mr. W. H. : «I could almost fancy that I saw him standing in the shadow of my room, so well had Shakespeare drawn $\operatorname{him}^{43} \gg(P W$ II : 159). Les deux figures gémellaires du dédicataire du recueil et du narrataire des Sonnets, et donc la masculinité, à travers ses deux mêmes figures idéalisées, se ramènent à une inscription textuelle, poème, dédicace énigmatique et jeux de mots, dont le langage demeure cryptique et facétieux, justifiant une lecture queer de l'identité.

On a vu combien la dynamique de la nouvelle reposait sur l'activité de lecture critique, et il faut lui appliquer son propre mode opératoire pour réévaluer la masculinité qui travaille le texte. La structure enchâssée doit pourtant être explicitée avant d'être interprétée. The Portrait of Mr.W. H. relate la tentative de retrouver l'identité du dédicataire des Sonnets, inscrite peut-être dans les poèmes, eux-mêmes décryptés par Cyril dans un récit soigneusement articulé, qu'il communique plus tard à Erskine. Ce dernier reprend la théorie de son ami, la reformule puis la dévoile au narrateur, qui finalement précise certains éléments et expose son raisonnement au lecteur. En d'autres termes, élaborer l'hagiographie jamais attestée de Mr. W. H. offre l'occasion à chacun d'eux de construire sa représentation d'un homme donné comme parfait. Le chiffre de l'idéal masculin circule, témoignage à préserver, à traduire en l'interprétant avant de le transmettre. Le tableau, sorte d'idéogramme, met le processus en abyme, puisqu'il recèle des éléments prélevés dans le legs encodé des Sonnets, mais porte les marques du désir de Cyril, matérialisé par les quelques gouttes de son propre sang projetées sur le nom de William Hughes (PW I : 151). À la mort de Cyril, l'œuvre picturale devient la propriété d'Erskine, qui meurt lui aussi, après s'être assuré qu'il reviendrait au narrateur de s'approprier ce portrait de la masculinité, cette masculinité conçue comme représentation, c'est-à-dire

41. « [R]iche en Désir / Will, à ton Désir / Will donne l'ampleur / d'un seul et mien désir / Will dont le tien s'élargisse. »

42. « $[U]$ n aspect d'homme/Hughes, où tous aspects/Hughes trouvent leur loi, ».

43. « J'ai eu l'impression de le voir, pour ainsi dire, se tenant dans l'ombre de ma chambre, tant Shakespeare l'avait dessiné avec soin. » 
comme récit. Chaque lecteur tente d'établir le sens de l'œuvre shakespearienne, afin de lever le mystère qui fonde sa propre identité d'homme.

Le fonctionnement de la nouvelle renverse cette logique et conduit les personnages à se produire à l'aide d'une autofiction, contestant l'idée d'authenticité, de spontanéité et d'adhérence au donné. William Hughes, s'il existe, y subit l'influence fictionnalisante d'autrui, puisqu'il doit se couler dans des rôles shakespeariens, qui seuls lui assurent une existence pleine et durable. En revanche, le Barde y choisit de se projeter dans la persona des poèmes, se forgeant une identité métamorphique au travers des situations d'énonciation qu'il imagine. Il en va de même pour Wilde vis-à-vis de ses personnages, et le cas de Cyril n'est pas différent. Il définit William Hughes dans sa théorie, détermine sa physionomie grâce au portrait qu'il fait peindre et finit par s'autoproduire en s'identifiant au narrataire des poèmes. On voit que ce motif doit beaucoup au principe de plaisir, tout en répondant à une conception potentiellement ludique du monde. S'aventurant dans les salons de The Importance of Being Earnest, où Algernon s'invente un ami bien utile nommé Bunbury, le Jaques de $A s$ You Like It énoncerait la formule d'un tel univers : «all the Bunburyist's world is a stage ${ }^{44}$. 》

Wilde prélève dans le recueil des éléments propres à construire un texte exclusivement homoérotique, mais il est possible d'inverser les logiques appropriatives. Alors, une conception alternative de la masculinité s'affirme, que le Shakespeare convoqué montre quelque réticence à sexualiser, et que Wilde prend soin de déconnecter d'un rapport aux femmes. Décontextualisant le concept d'amour amical de la Renaissance, ou bien le recontextualisant, la nouvelle met en scène des personnages, pourtant d'âge respectable, qui ne sont apparemment pas mariés. Ils ne s'intéressent visiblement pas à l'exultation physique, menant une vie d'esthète, recherchant dans des plaisirs sophistiqués les sensations propres à les satisfaire. Se dessine l'image d'une masculinité post-génitale qui ne doit pas être confondue avec une forme de fixation prégénitale. Il est bien sûr possible d'évoquer l'action conjuguée de la censure et du refoulement, qui entraînent une abstinence empreinte de frustration. Morale en son fond, cette condamnation ne perçoit pas la volonté d'émancipation vis-à-vis des logiques hétérosexuelles et même des identifications sexuelles. Réinvestissant sa libido dans les sphères qu'elle se choisit, sans état d'âme, selon une logique aristocratique qui pose sans démontrer, cette masculinité, tout comme l'existence en général, se donne des valeurs qui n'obéissent pas à un régime de la preuve. Wilde montre d'ailleurs que si le tableau de William Hughes n'est pas authentique, il oriente authentiquement l'existence de Cyril et lui assure la vie pleine que chacun peut souhaiter.

44. «Le monde du Bunburyist est tout entier une scène de théâtre. » 
Bien que The Portrait of $M r . W . H$. semble d'abord remplir, vis-à-vis des Sonnets, une fonction muséale, l'originalité de la démarche est mise au service d'une stratégie de rupture. En outre, le concept du critique wildien encourage à reconfigurer le message de la nouvelle, afin de contribuer à affirmer des points de vue singuliers. Dans la situation d'énonciation qui est la leur, ces postulats contribuent à faire entendre de façon polyphonique les accents d'une masculinité plurielle, en mutation, sensible à tous les types d'intervention. D'ailleurs, la fin ouverte invite indirectement le lecteur à se lancer dans l'étude interprétative de la théorie présentée : «I think that there is really a great deal to be said for the Willie Hughes theory of Shakespeare's Sonnets ${ }^{45} »(P W$ III : 169). La réalité avait inspiré la littérature, qui la transforme en retour, et la formulation to be said, "à dire ", projette le dispositif dans l'avenir extradiégétique. De façon similaire, William Hughes avait selon Wilde influencé la poésie et l'art dramatique de Shakespeare, qui contaminent ensuite l'existence des personnages wildiens, avant que leur récit ne quitte son cadre natif pour coloniser le réel, mais aussi pour en révéler la part fictionnelle.

Gilbert Pham-Thanh

Université Paris $13-$ CENEL

45. « Je pense qu'il y aurait beaucoup à dire en faveur de la théorie relative à Willie Hughes pour comprendre les Sonnets de Shakespeare ». 